\title{
VEGF-A stimulates lymphangiogenesis and hemangiogenesis in inflammatory neovascularization via macrophage recruitment
}

\author{
Claus Cursiefen, ${ }^{1}$ Lu Chen, ${ }^{1}$ Leonardo P. Borges, ${ }^{1}$ David Jackson, ${ }^{2}$ Jingtai Cao, ${ }^{3}$ \\ Czeslaw Radziejewski, ${ }^{3}$ Patricia A. D'Amore, ${ }^{1}$ M. Reza Dana, ${ }^{1}$ \\ Stanley J. Wiegand, ${ }^{3}$ and J. Wayne Streilein ${ }^{1}$
}

${ }^{1}$ The Schepens Eye Research Institute, Department of Ophthalmology, Harvard Medical School, Boston, Massachusetts, USA. 2MRC Human Immunology Unit, Institute of Molecular Medicine Oxford, Oxford, United Kingdom. ${ }^{3}$ Regeneron Pharmaceuticals Inc., Tarrytown, New York, USA.

\begin{abstract}
Lymphangiogenesis, an important initial step in tumor metastasis and transplant sensitization, is mediated by the action of VEGF-C and -D on VEGFR3. In contrast, VEGF-A binds VEGFR1 and VEGFR2 and is an essential hemangiogenic factor. We re-evaluated the potential role of VEGF-A in lymphangiogenesis using a novel model in which both lymphangiogenesis and hemangiogenesis are induced in the normally avascular cornea. Administration of VEGF Trap, a receptor-based fusion protein that binds and neutralizes VEGF-A but not VEGF-C or -D, completely inhibited both hemangiogenesis and the outgrowth of LYVE-1 $1^{+}$lymphatic vessels following injury. Furthermore, both lymphangiogenesis and hemangiogenesis were significantly reduced in mice transgenic for VEGF-A ${ }^{164 / 164}$ or VEGF-A ${ }^{188 / 188}$ (each of which expresses only one of the three principle VEGF-A isoforms). Because VEGF-A is chemotactic for macrophages and we demonstrate here that macrophages in inflamed corneas release lymphangiogenic VEGF-C/VEGF-D, we evaluated the possibility that macrophage recruitment plays a role in VEGF-A-mediated lymphangiogenesis. Either systemic depletion of all bone marrow-derived cells (by irradiation) or local depletion of macrophages in the cornea (using clodronate liposomes) prior to injury significantly inhibited both hemangiogenesis and lymphangiogenesis. We conclude that VEGF-A recruitment of monocytes/macrophages plays a crucial role in inducing inflammatory neovascularization by supplying/amplifying signals essential for pathological hemangiogenesis and lymphangiogenesis.
\end{abstract}

\section{Introduction}

Angiogenesis, the outgrowth of new from preexisting blood vessels, is an important pathogenic aspect of tumor growth, chronic inflammatory diseases, and most blinding ocular conditions (for review see ref. 1). To clearly separate it from the process of lymphangiogenesis, we will refer to blood vascular angiogenesis as hemangiogenesis (HA). In recent years, much has been learned about the stimulators and inhibitors of HA and lymphangiogenesis, and members of the VEGF family have emerged as prime mediators of both processes (for review see refs. 2-4). The VEGF growth factor family consists of five members that bind to and activate three distinct receptors. VEGF-A binds to VEGFR1 and VEGFR2, and placental growth factor (PIGF) and VEGF-B bind only to VEGFR1. VEGF-C and VEGF-D bind to VEGFR2 and VEGFR3 (for review see ref. 2).

VEGF-A has clearly emerged as the family member principally responsible for normal vasculogenesis and HA. The direct effects of VEGF-A on vascular endothelial cells are mediated principally

Nonstandard abbreviations used: chorioallantoic membrane (CAM); corneal neovascularization (CNV); hemangiogenesis (HA); placental growth factor (PlGF); platelet-endothelial cell adhesion molecule 1 (PECAM-1); resonance unit (RU).

Conflict of interest: J. Cao, C. Radziejewski, and S.J. Wiegand are employees of Regeneron Pharmaceuticals Inc.

Citation for this article: J. Clin. Invest. 113:1040-1050 (2004). doi:10.1172/JCI200420465. via VEGFR2 ligation, while, until recently, VEGFR1 was thought to mediate mainly inhibitory or decoy functions (for review see refs. 1, 2). VEGF-A also plays a predominant role in diverse forms of pathological angiogenesis, including those requisite for the rapid growth of solid tumors (for review see refs. 1,2). For this reason many antiangiogenic agents currently in development for the treatment of cancers have targeted VEGF-A or VEGFR2 (for review see refs. 2, 3; http://www.cancer.gov).

In contrast to HA, lymphangiogenesis is thought to be mediated mainly by the binding of VEGF-C and -D to their high-affinity receptor, VEGFR3 (for review see ref. 4). Like HA, lymphangiogenesis has gained much attention recently as an important initial step in tumor pathogenesis (for review see ref. 4; refs. 5-7). It has been shown that intra- and/or peritumoral lymphangiogenesis increases the risk for metastasis both in animal models and in human tumors (for review see ref. 4). The release of the lymphangiogenic growth factors VEGF-C and -D has been linked to a circulating subfraction of CD14+, VEGFR3-expressing monocytes that are recruited to and activated at the site of tumor growth (8). Antilymphangiogenic strategies targeting VEGFR3-mediated signaling have been reported to inhibit lymphangiogenesis and improve survival in animal models of metastatic cancer (5).

As noted above, VEGF-C and -D also bind to VEGFR2 and display hemangiogenic activities in certain situations $(9,10)$. In contrast, VEGF-A is thought to act solely as a hemangiogenic 
factor, as placement of VEGF-A-impregnated pellets in the cornea (11), overexpression of VEGF-A in the skin (12-14), and VEGF-A applied to the chorioallantoic membrane (15) have all been reported to cause HA but not lymphangiogenesis. However, it has recently been shown that, like blood endothelial cells, lymphatic endothelial cells also express VEGFR2, and VEGF-A potently promotes their survival in vitro (16-19). Moreover, adenoviral overexpression of VEGF-A in the rabbit ear leads to the formation of hyperplastic, "giant" lymphatic vessels, further suggesting that VEGF-A has the potential to stimulate some forms of lymphangiogenesis (20).

As several antiangiogenic agents that target VEGF-A have already entered clinical testing, the question of whether such agents might also affect lymphangiogenesis has taken on particular importance (4-7). In addition to promoting tumor metastases, induction of lymphangiogenesis is also associated with the termination of the immune-privileged state of the normally avascular cornea. The significant deterioration of corneal transplant survival under these conditions makes it imperative to determine whether antiangiogenic strategies that target VEGF-A also interfere with corneal lymphangiogenesis (for review see ref. 21).

To address this question and to resolve conflicting findings regarding the role of VEGF-A in lymphangiogenesis, we first characterized a novel model of inflammatory neovascularization in the cornea to determine whether HA is accompanied by lymphangiogenesis $(22,23)$. We then evaluated the effect of selectively blocking the actions of endogenous VEGF-A (and PlGF) using VEGF Trap (24) or of altering endogenous VEGF-A expression by using transgenic mice that express only VEGF-A isoform 164 or 188 (VEGF-A ${ }^{164 / 164}$ or VEGF-A ${ }^{188 / 188}$, respectively) $(25,26)$. Finally, as VEGF-A is known to recruit VEGFR1-expressing monocytes/ macrophages $(27,28)$, which are known to release not only hemangiogenic but also lymphangiogenic growth factors (8), and as VEGF-mediated HA and lymphangiogenesis in our model was accompanied by a marked inflammatory response, we evaluated (a) systemic depletion of bone marrow-derived cells and (b) local depletion of macrophages for their effects on lymphangiogenesis and HA following corneal injury.

\section{Methods}

Mice and anesthesia. The generation of knock-in mice expressing only VEGF-A isoform 164 or 188 on a Swiss Webster background has been described previously $(25,26)$. BALB/c mice $6-8$ weeks of age were used in all experiments not involving knock-in mice (Taconic Farms, Germantown, New York, USA). All mice examined were between 8 and 12 weeks of age and were treated in accordance with the Association for Research in Vision and Ophthalmology Statement for the Use of Animals in Ophthalmic and Vision Research. Mice were anesthetized using a mixture of ketamine and xylazine $(120 \mathrm{mg} / \mathrm{kg}$ body weight and $20 \mathrm{mg} / \mathrm{kg}$ body weight, respectively).

Mouse model of suture-induced, inflammatory corneal neovascularization. The mouse model of suture-induced inflammatory corneal neovascularization (CNV) was used as previously described (29). Briefly, a 2-mm-diameter corneal trephine was placed gently on the central cornea of anesthetized mice solely to mark the central corneal area. Three 11-0 sutures were then placed intrastromally with two stromal incursions each extending over $120^{\circ}$ of the corneal circumference. The outer point of suture placement chosen was halfway between the limbus and the line outlined by the $2-\mathrm{mm}$ trephine; the inner suture point was at the same distance from the $2-\mathrm{mm}$ trephine line to obtain standardized angiogenic responses. Sutures were left in place for 7 days. Mice were euthanized and the cornea with limbus was excised, and flat-mount double-immunohistochemistry was performed as described below.

Immunobistochemistry and morphometry of $H A$ and lymphangiogenesis in the cornea. Briefly, corneal flat mounts were rinsed in PBS, fixed in acetone, rinsed in PBS, blocked in 2\% BSA, stained with FITCconjugated CD31 (platelet-endothelial cell adhesion molecule 1 [PECAM-1]) antibody overnight (1:100 dilution; Santa Cruz Biotechnology, Santa Cruz, California, USA), washed, blocked, and stained with anti-LYVE-1 (1:500 dilution; LYVE-1 is a lymphatic endothelium-specific hyaluronic acid receptor; D. Jackson, Oxford University, Oxford, United Kingdom) (22,30), which was visualized using a indocarbocyanine-conjugated secondary antibody (1:100 dilution; Jackson ImmunoResearch Laboratories, Westgrove, Pennsylvania, USA). Double-stained sections were analyzed using a Zeiss Axiophot microscope. Digital pictures of the flat mounts were obtained using the Spot Image Analysis system (Spectra Services Inc., Webster, New York, USA), and the area covered by CD $31^{+++}$LYVE-1- blood vessels and CD $31^{+}$LYVE- $1^{+++}$lymph vessels $(22,23)$ (where +++ indicates strong positivity; ++, medium positivity; and +, mild positivity) was measured using NIH Image software. The total corneal area was outlined using the innermost vessel of the limbal arcade as the border, and the area of blood and lymphatic neovascularization within the cornea was then calculated and normalized to the total corneal area (expressed as a percentage of the cornea covered by vessels). Paraffin embedding of corneas and immunostaining for LYVE-1 and counterstaining with hematoxylin and eosin was done as described previously (22).

Histological characterization and quantification of inflammatory cells and immunohistochemistry for VEGF-C and VEGF-D. The presence of inflammatory cells in normal corneas and their recruitment into corneas 1 week after suture placement was quantified in hematoxylin and eosin-stained serial sections of plastic-embedded corneas fixed in $10 \%$ paraformaldehyde after enucleation. In addition, for further characterization of inflammatory cells recruited to the cornea, double immunohistochemistry was performed on corneal whole mounts and frozen sections with the macrophage markers CD11b (Pharmingen, San Diego, California, USA), CD68 (Santa Cruz Biotechnology), and F4/80 (Caltec, San Francisco, California, USA), the panleukocyte marker CD45 (Pharmingen) and the neutrophil marker GR1 (Pharmingen) as described previously (22).

For identification of the intracorneal source of lymphangiogenic growth factors VEGF-C and -D, double immunohistochemistry for VEGF-C and -D (polyclonal antibody; 1:100 dilution; Santa Cruz Biotechnology) and the macrophage markers mentioned above was performed on corneal whole mounts 48 hours after corneal suture placement with additional Fc blockade (Santa Cruz Biotechnology). Sections were evaluated using confocal microscopy (Leica TCS - SP2 Confocal Laser Scanning Microscope, Leica, Wetzlar, Germany).

Selective neutralization of VEGF-A and PlGF using VEGF traps. VEGF TrapR1R2 is a fusion protein comprising portions of the extracellular domains of human VEGFR1 (IgG domain 2) and VEGFR2 (IgG domain 3) coupled to the Fc portion of human IgG1 (Regeneron Pharmaceuticals Inc, Tarrytown, New York, USA) (24), VEGF Trapr1R2 selectively binds VEGF-A and PlGF but not VEGF-C/ 
VEGF-D (see below). Mice received a single injection of VEGF TrapR1R2 intraperitoneally at a dose of $12.5 \mathrm{mg} / \mathrm{kg}$ at time of corneal injury. Control mice received an injection of human Fc $(12.5 \mathrm{mg} / \mathrm{kg}$ intraperitoneally). In one study, we used another Trap (VEGF TrapR1/A40) that comprised only portions of VEGFR1 (IgG domains 1-3) fused to Fc to completely obviate the possibility of binding to VEGF-C and -D. As this construct exhibits reduced bioavailability as well as lower affinity for binding of VEGF-A compared with the VEGF Trap 1 R2, it was administered at a dose of 25 $\mathrm{mg} / \mathrm{kg}$ (intraperitoneally).

Biochemical characterization of binding of VEGF-A, -C, and -D to VEGF $T_{\text {Trap }}$ RIR $2_{2}$ and VEGF Trap $R 1 / A 40$. The specificity of binding of VEGF family members to various VEGF receptor chimeras was assessed using Biacore (Biacore, Piscataway, New Jersey, USA). Protein A (Pierce Biotechnology, Rockford, Illinois, USA) was amine-coupled (2000 resonance units [RU]) onto CM5 chips on all flow cells, and VEGF TrapR1R2 and VEGF TrapR1/A40 were captured onto the chip surface at levels of 1,324 and 2,315 RU, respectively. VEGFR1-Fc, VEGFR2-Fc, and VEGFR3-Fc (R\&D Systems, Minneapolis, Minnesota, USA) were used as control proteins; these constructs comprise the full extracellular domain of the indicated human receptor fused to human $\mathrm{Fc}$ and were captured on protein A-coated chips at 530, 522 and 441 RU. A flow cell with only amine-coupled protein A was used to allow subtraction of nonspecific binding.

VEGF ligands (325 $\mu \mathrm{l}$ each) were injected at a rate of $10 \mu \mathrm{l} / \mathrm{min}$ in HEPES saline buffer containing $0.1 \mathrm{mg} / \mathrm{ml}$ of BSA (Fluka, Buchs, Switzerland). Human VEGF-C, human VEGF-D, and mouse VEGF-D (all from R\&D) were injected at a concentration of 200 nM each. Human VEGF-A ${ }^{165}$, human VEGF-A ${ }^{121}$ (Regeneron Pharmaceuticals Inc.) and mouse PlGF-2 (R\&D) were injected at a concentration of $50 \mathrm{nM}$ each. Two 1-minute pulses of $100 \mathrm{mM}$ $\mathrm{H}_{3} \mathrm{PO}_{4}$ were used to clean protein A surfaces, and receptor-Fc chimeras were recaptured on the chip for each ligand evaluated. Data were expressed as RU of specifically bound ligand per femtomole of receptor fusion protein captured on the protein A surface.

Analysis of lymphangiogenic effects of VEGF-A and VEGF-C in the corneal micropocket assay. The corneal micropocket assay was performed as previously described (10). Briefly, corneal micropockets were created using a modified von Graefe knife, and a micropellet $(0.4 \times 0.4 \mathrm{~mm})$ of sucrose aluminum sulfate coated with hydron polymer containing $200 \mathrm{ng}$ of VEGF- $\mathrm{A}_{164}(\mathrm{R} \& \mathrm{D})$ or $200 \mathrm{ng}$ of recombinant rat VEGF-C as a positive control (RDI, Flanders, New Jersey, USA) was implanted into each pocket. The pellet was positioned $0.6-0.8 \mathrm{~mm}$ from the limbus and the site was covered with antibiotic ointment (erythromycin) and was left in place for 10 days ( $n>10$ mice each). Hemangiogenic and lymphangiogenic responses were quantified as described above using double immunostaining with CD31/LYVE-1. The maximal extent of blood versus lymph vessel outgrowth between subjacent limbus and pellet was graded semiquantitatively in four categories for both vessel types: 0 , no outgrowth; 1 , outgrowth less than $1 / 3$ of the limbuspellet distance; 2 , outgrowth between $1 / 3$ and $2 / 3$ of the limbus-pellet distance; 3 , vessel reaching pellet.

Systemic depletion of bone marrow-derived cells by $\gamma$-irradiation of mice. BALB/c mice were pretreated with acidified water for 3 days and then were exposed to a single dose of 9 Gy whole body $\gamma$-irradiation. After 18 hours, sutures were placed into the corneas as described above. Control mice received the acidified water pretreatment and suture placement. Seven days later, the mice were euthanized and their corneas were removed for flat-mount staining and morphometry as described above (at least three mice per group per experiment).

Local depletion of macrophages using subconjunctival clodronate liposomes. Local depletion of monocytes/macrophages was accomplished as described previously $(31,32)$. Liposomes filled with dichloromethylene diphosphonate (CL2MDP-LIP; $10 \mu \mathrm{l}$; a generous gift from Nico van Rooijen, Vrije Universiteit, Amsterdam, The Netherlands) was injected subconjunctivally at the time of suture placement and 2, 4, and 6 days after surgery. Control group mice received either liposomes containing PBS or only PBS subconjunctivally at the same time points. To rule out the possibility of vascular endothelial uptake of clodronate liposomes and a direct effect of clodronate on blood and lymphatic endothelium, the effect of clodronate versus Fc protein injected subconjunctivally on preexisting pathological corneal vessels (previously induced by corneal suturing) and normal limbal vessels was assessed 12 hours after injection.

Cultivation of bone marrow-derived macrophages. Bone marrowderived macrophages were harvested and cultured as previously described (33). Briefly, BALB/c mice 6 weeks of age were euthanized, their femur bones were dissected and cut at both ends, and the bone marrow was flushed into HBSS (Cambrex Bio Science, Verviers, Belgium) using a PBS-filled 25-gauge needle. Then, the bone marrow cells were washed and resuspended in growth medium consisting of DMEM (Sigma-Aldrich, St. Louis, Missouri, USA) with 10\% horse serum (Sigma-Aldrich), 10\% CPSR-1 (Sigma-Aldrich), 10\% L929 cell-conditioned medium, 1\% MEM vitamins (Invitrogen, Carlsbad, California, USA), 1\% sodium pyruvate (Cambrex), 1\% NEAA (Cambrex), 1\% L-glutamine (Cambrex), and $1 \%$ penicillin/streptomycin (Cambrex).

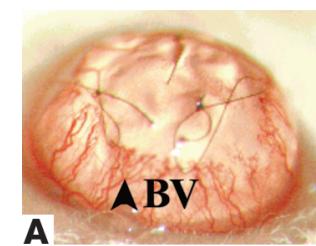

A

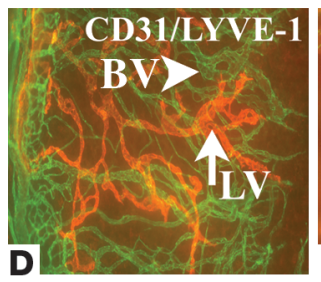

Figure 1

Concomitant induction of $\mathrm{HA}$ and lymphangiogenesis in inflammatory corneal neovascularization. (A-F) Seven days after central, intrastromal suture placement $(\mathbf{A})$, a robust angiogenic response (A; blood vessel $[\mathrm{BV}])$ in combination with an influx of inflammatory cells (B [H\&E] and $\mathbf{C}$ ) can be seen biomicroscopically (A) and by using CD31 (PECAM1) immunostaining (D) of corneal flat mounts (green). The CD45+ inflammatory cell infiltrate (C) consists mainly of GR-1+ neutrophils (red) and F4/80+ macrophages (green). In addition to the CD $31^{+++}$LYVE-1- blood vessels ( $D$ and $\mathbf{E}$; green), there is parallel outgrowth of CD31+LYVE-1+++ lymphatic vessels (LV; D-F; red). Blood vessels do not react with the lymphatic vascular-specific hyaluronic acid receptor LYVE-1 (F). Magnification, $\times 20(\mathbf{A}), \times 200(\mathbf{B}$ and F), $\times 400(\mathbf{C}$ and E), and $\times 100$ (D). 

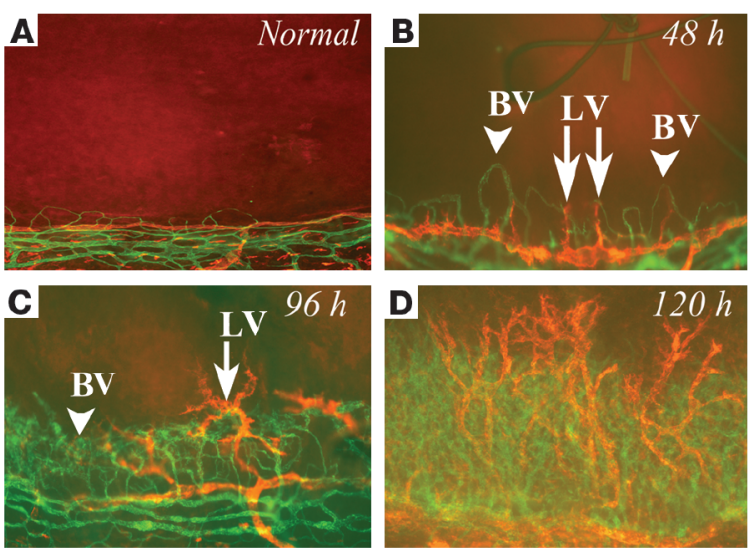

\section{Figure 2}

Time course of early inflammatory HA and lymphangiogenesis. (A-D) In inflammatory corneal neovascularization, there is very early and parallel outgrowth of both blood vessels (green) as well as lymphatic vessels (red) from the limbal vascular arcade (bottom of each picture) toward the suture into the normally avascular cornea (top of each picture). Both vessel types sprout as early as 24 hours after injury and progress over time, with lymphatic vessels (red staining) often preceding blood vessels (green staining). Magnification, $\times 100$.
After 7 days of culture, adherent cells were then processed for RNA and RT-PCR as described above.

Statistical analysis. Statistical significance was analyzed by the MannWhitney $U$ test. Differences were considered significant at $P<0.05$. Each experiment was performed at least twice with similar results. Graphs were drawn using Graph Pad Prism, Version 3.02 (Graph Pad Software, San Diego, California, USA).

\section{Results}

Suture-induced, inflammatory CNV is characterized by HA, lymphangiogenesis, and inflammatory cell infiltration. To address the question of whether endogenous VEGF-A might be involved in lymphangiogenesis, we first studied an established model of suture-induced inflammatory CNV to evaluate the outgrowth of lymphatic vessels into the normally avascular cornea $(22,29)$. This model is characterized by a robust outgrowth of new blood vessels from the limbal arcade (Figure 1, A-C) and is routinely used in the mouse to create a vascularized "high-risk bed" for corneal transplantation studies. New blood vessels reached the sutures at 1 week after surgery and were accompanied by a dense inflammatory cell infiltrate. CD $45^{+}$inflammatory cells within the corneal stroma mainly consisted of $\mathrm{GR}-1^{+}$neutrophils and, less prominently, also $\mathrm{F} 4 / 80^{+} \mathrm{CD} 11 \mathrm{~b}^{+}$macrophages (Figure 1 ). To determine whether this early HA was accompanied by lymphangiogenesis, corneal whole mounts were double-stained using CD31 as a panendothelial marker and LYVE-1 $(22,30)$ as specific lymphatic vessel marker. One week after surgery, both CD $31^{+}$LYVE- $1^{+++}$lymphatic vessels as well as CD $31^{+++}$LYVE-1- blood vessels grew into the cornea (Figure $1, \mathrm{D}-\mathrm{F})$, demonstrating that a robust lymphangiogenesis is also induced in this CNV model.

\section{Figure 3}

Neutralization of VEGF-A inhibits HA and lymphangiogenesis. (A-F) A molecular trap designed to bind VEGF-A (VEGF Trap R1R2) completely inhibits both HA and lymphangiogenesis within 1 week after injury. Whereas mice receiving an intraperitoneal injection of Fc protein at surgery (Fc control) display robust angiogenesis (A, slit-lamp picture; B, CD31 staining) and lymphangiogenesis (C, CD31 and LYVE-1 staining) 1 week later, mice treated with a single injection of VEGF Trap $\mathrm{R}_{\mathrm{R} 1 \mathrm{R} 2}$ do not show HA ( $\mathbf{D}$ and $\mathbf{E}$; blood vessels are green) or lymphangiogenesis (F; lymph vessels are red). Magnification, $\times 100(\mathbf{C}-\mathbf{F})$. (G) Morphometric analysis of the nearly complete inhibitory effect of VEGF Trap on both $\mathrm{HA}$ and lymphangiogenesis $(P<0.001)$. Magnification $(\mathbf{A}$ and $\mathbf{B}), \times 20$.
Blood and lymphatic vessels display rapid and parallel outgrowth in CNV. Based on wound healing studies in skin, it has been suggested the ingrowth of lymphatic vessels is delayed for several days relative to that of blood vessels (34). To determine whether this holds true for the CNV model, we conducted a time-course study comparing the outgrowth of both vessel types. As is illustrated in Figure 2, HA and lymphangiogenesis occurred contemporaneously. Small sprouts arising from pre-existing limbal vessels could be detected as early as 24 hours after surgery, and outgrowth of new vessels of both types was clearly visible at 48 hours (Figure 2). Interestingly, lymphatic vessels sometimes grew in advance of blood vessels at the leading edge of growth (Figure 2).
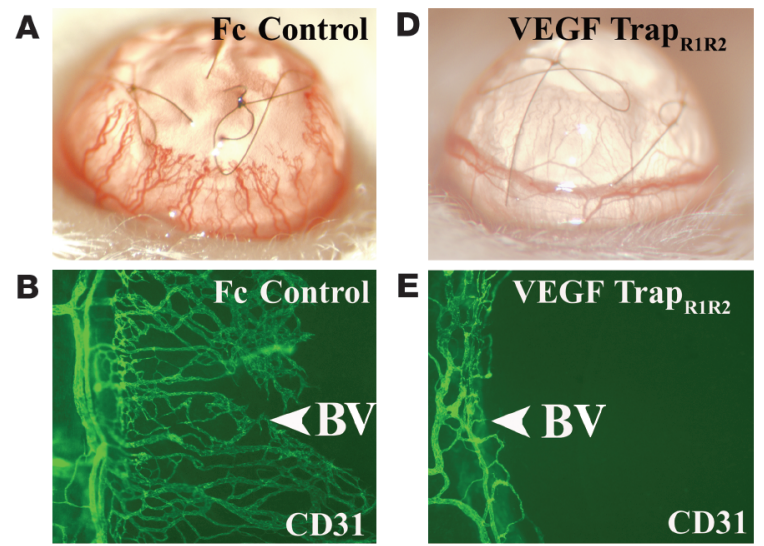

C
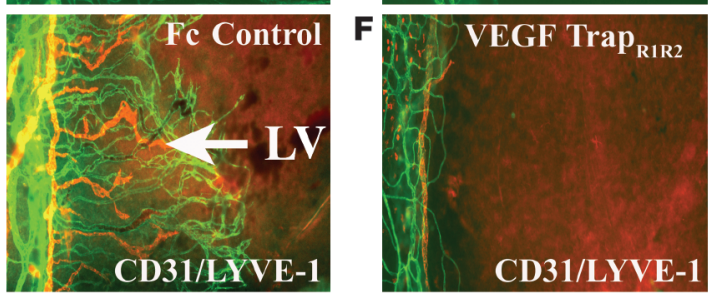

G

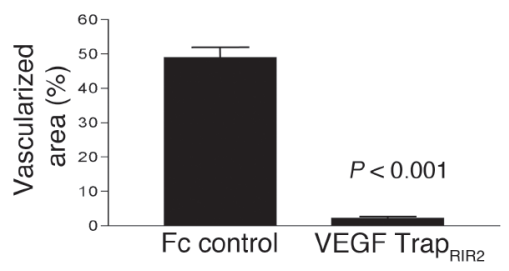




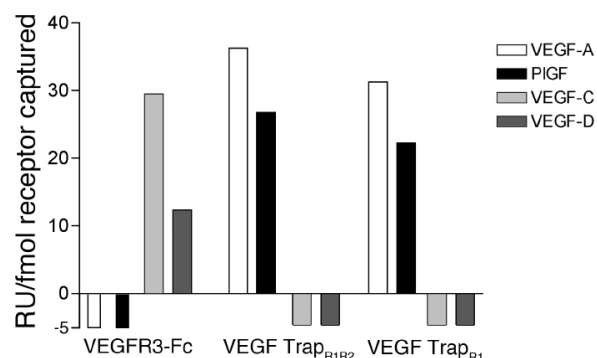

Administration of VEGF Trap R1R2 $_{2}$ completely inbibits HA and lymphangiogenesis in inflammatory $C N V$. To determine the extent to which VEGF-A is important for inflammation-associated lymphangiogenesis, we treated mice systemically with a molecular trap that selectively binds and neutralizes VEGF-A but not VEGF-C or -D (VEGF Trap $p_{R 1 R 2}$ ). Administration of VEGF-Trap 1 R2 completely prevented both HA and lymphangiogenesis 7 days after suture placement, as determined by examination of the corneas (Figure 3; the area vascularized by blood and lymphatic vessels was $49 \% \pm 12 \%$ in Fc-treated mice and $2.3 \% \pm 1.5 \%$ in mice treated with VEGF $\left.\operatorname{Trap}_{\mathrm{R} 1 \mathrm{R} 2}, P<0.001\right)$. Moreover, examination of corneas at days 2,4 , and 7 after suture placement revealed that blood and lymphatic vessels never grew out from the limbus in the Trap-treated group.

Although in vitro binding studies showed that VEGF TrapR1R2 binds only VEGF-A and PlGF with high affinity, but not VEGF-C or -D (see below), we further ruled out the possibility that the observed response might be due to neutralization of VEGF-C and -D in vivo by repeating the above experiment using VEGF Trap $_{\mathrm{R} 1 / \mathrm{A} 40}$. Though it is less bioavailable and exhibits a lower affinity for VEGF-A, this reagent consists only of the ligand-binding domain of VEGFR1 but not VEGFR2, and thus it is inherently incapable of binding VEGF-C or -D. Using this agent we observed a similar parallel and significant, albeit less complete, inhibition of both HA $(53.8 \% \pm 14.6 \%$ versus $23.6 \% \pm 6.8 \%)$ and lymphangiogenesis $(45.7 \% \pm 15.6 \%$ versus $26 \% \pm 8.2 \%$; $P<0.05)$.

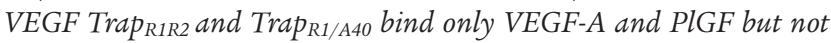
$V E G F-C / V E G F-D$. When added to tissue cultures at approximately equimolar concentrations, VEGF TrapR1R2 has been shown to block VEGF-A-induced phosphorylation of VEGFR2 as well as proliferation of primary human umbilical vein endothelial cells (24). VEGF TrapR1R2 $_{2}$ binds $V_{E G F}$ 165 with very high affinity $\left(\mathrm{K}_{\mathrm{D}}, \sim 1 \mathrm{pM}\right)$. Similar results have been obtained using murine $\mathrm{VEGF}_{164}$, and in preliminary studies mouse PlGF was also found to bind to VEGF Trap $_{R 1 R 2}$ with high affinity $\left(\mathrm{K}_{\mathrm{D}}, \sim 1.8 \mathrm{pM}\right)$. The results of Biacore binding studies confirmed that both VEGF TrapR1R2 and Trap 1 R/A40 selectively bound VEGF-A (VEGF 165 and VEGF $_{121}$ ) and PlGF, but there was no detectable binding of VEGF-C or -D to either VEGF Trap at concentrations up to $200 \mathrm{nM}$ (Figure 4 and Table 1). VEGFR1-Fc demonstrated the same pattern of binding to the above VEGF family members. In contrast, VEGFR3-Fc avidly bound VEGF-C and -D but not PlGF or either isoform of VEGF-A. Collectively, these data clearly demonstrate

\section{Table 1}

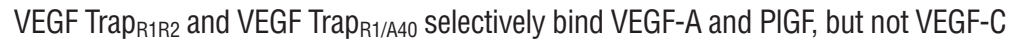
and VEFG-D

that VEGF TrapR1R2 and VEGF TrapR1/A40 bind only VEGF-A and PlGF but not VEGF-C or -D, whereas VEGFR3-Fc binds only VEGF-C and -D but not VEGF-A or PlGF.

Mice that express only VEGF- $A_{188}$ or VEGF- $A_{164}$ display significantly reduced $H A$ and lymphangiogenesis. To further evaluate the role of VEGF-A in promoting lymphangiogenesis, we studied mice that express only VEGF-A isoform 164 or 188 . We hypothesized that specific genetic deletion of VEGF-A isoforms should only affect lymphangiogenesis if VEGF-A is involved in mediating lymphangiogenesis. Sutures were placed in the corneas; 1 week later, VEGF ${ }^{164 / 164}$ mice (lacking VEGF-A isoforms 120 and 188) displayed an area of HA of $27.9 \% \pm 12 \%$ and an area of lymphangiogenesis of $22.7 \% \pm 13.6 \%$. Sutured corneas of VEGF ${ }^{188 / 188}$ transgenic animals (lacking VEGF-A isoforms 120 and 164) displayed an area of $\mathrm{HA}$ of $20.3 \% \pm 10 \%$ and an area of lymphangiogenesis of $25 \% \pm 12.7 \%$. These represent significant reductions in areas of both lymphangiogenesis and HA compared with wild-type controls (HA, 44\% $\pm 10.2 \%$; area of lymphangiogenesis, $57.2 \% \pm 9.6 \%$; $P<0.05$; Figure 5). Thus, both HA and lymphangiogenesis can occur in the absence of VEGF-A isoforms 120 and 188 as well as in the absence of isoforms 120 and 164 . However, under these circumstances the extent of both corneal HA and lymphangiogenesis is equivalently diminished, suggesting that an orchestrated action of VEGF-A isoforms is necessary for lymphangiogenesis.

VEGF- $A_{164}$ can induce lymphangiogenic as well as hemangiogenic responses in the corneal micropocket assay. To determine whether exogenous VEGF-A can exert a direct lymphangiogenic effect, we studied the effect of VEGF- $\mathrm{A}_{164}$ in the corneal micropocket assay. Lymphangiogenesis as well as HA was induced in 17 of 20 corneas that had been implanted with pellets $(200 \mathrm{ng})$ of VEGF-A. Lymphatic vessels were noted to be appreciably shorter than the accompanying blood vessels (semiquantitative grading, $2.7 \pm 0.7$ versus $1 \pm 0.9 ; P<0.01$; Figure 6 ). These findings indicate that VEGF-A alone can induce lymphangiogenesis, although less 

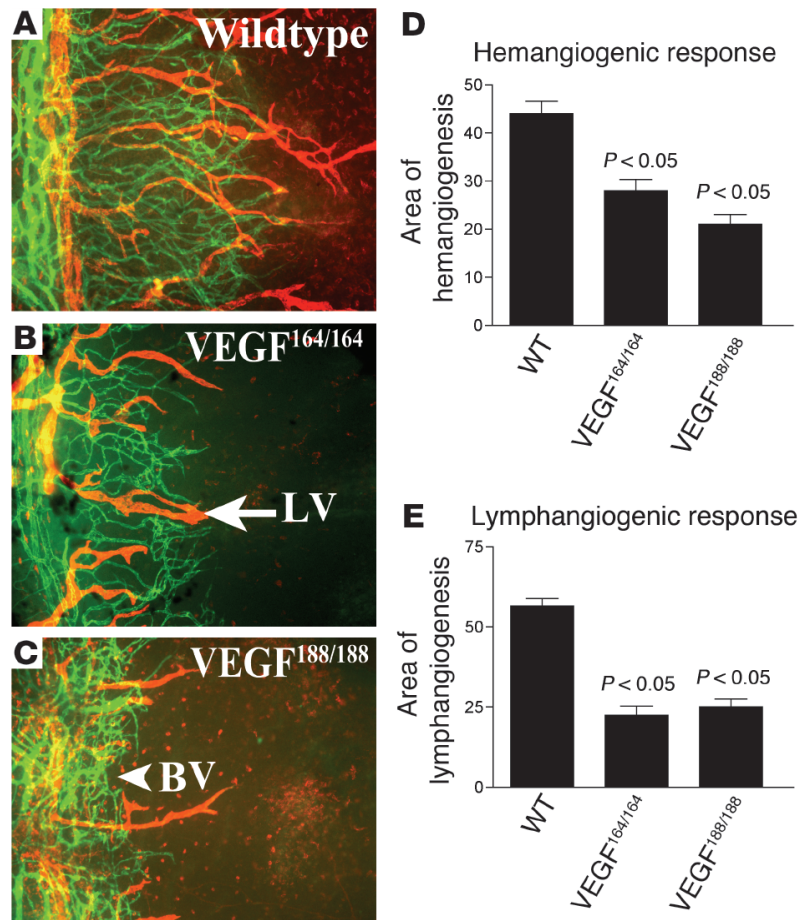

robustly than HA. The same was found for VEGF-C pellets used as controls. There was no significant difference in the ratio of HA versus lymphangiogenesis between VEGF-C and -A pellets $(P=0.8)$.

VEGF Trap RIR2 $_{2}$ significantly reduces the recruitment of inflammatory cells into the cornea. Because VEGF is chemotactic for inflammatory cells, for example, monocytes/macrophages via VEGFR1 $(27,28)$, and because macrophages can potentially secrete lymphangiogenic factors such as VEGF-C and -D $(8,35)$, we investigated whether neutralization of VEGF-A using VEGF TrapR1R2 would also impair the recruitment of bone marrow-derived cells into the cornea following suture injury. Animals that received a single intraperitoneal injection of VEGF TrapR1R2 at time of surgery exhibited significantly reduced numbers of stromal inflammatory cells compared with controls (Figure 7); the number of inflammatory cells per corneal cross-section in VEGF TrapR1R2-treated mice was $188 \pm 14$ compared with $909 \pm 167$ in Fc-treated control mice $(P<0.01)$. The inflammatory infiltrate in the Fc-treated controls was composed of GR-1 $1^{+}$neutrophils and, less often, F4/80+ macrophages.

\section{Figure 5}

Importance of VEGF-A isoforms for lymphangiogenesis. (A-E) Double immunostaining CD31/LYVE-1 (blood vessels, green; lymphatic vessels, red) of corneal flat mounts of wild-type mice (A), VEGF-A ${ }^{164 / 164}$ transgenic mice $(B)$, and VEGF-A ${ }^{188 / 188}$ transgenic mice (C) demonstrates significantly reduced $\mathrm{HA}(\mathbf{D} ; P<0.05)$ and lymphangiogenesis $(\mathbf{E} ; P<0.05)$. Magnification, $\times 100(\mathbf{A}-\mathbf{C})$.

Depletion of bone marrow-derived cells by whole-body $\gamma$-irradiation inhibits lymphangiogenesis. Macrophages can be recruited to sites of inflammation by VEGF-A via VEGFR1 interactions $(27,28)$, and activated macrophages are known to express a variety of cytokines and growth factors, including VEGF-A, -C, and -D $(8,35)$. Since the inhibition of corneal neovascularization by the VEGF Trap 1 R2 was associated with a marked decrease in the recruitment of inflammatory cells into the cornea, we determined whether depletion of inflammatory cells by other means would also inhibit HA and lymphangiogenesis following corneal injury. In preliminary experiments we confirmed that whole-body irradiation with a single dose of 9 Gy caused nearly complete depletion of leucocytes from the peripheral blood within 1 week of irradiation (data not shown). The results in Figure 8 show that depletion of bone marrow-derived cells by irradiation substantially inhibited both HA and lymphangiogenesis in response to corneal inflammatory stimuli. The areas of blood and lymphatic vessels in irradiated mice were $18.4 \% \pm 4 \%$ and $16.4 \% \pm 3.2 \%$, respectively, compared with $49.6 \% \pm 10.4 \%$ and $38 \% \pm 12.23 \%$ for blood and lymph vessels, respectively, in unirradiated controls $(P<0.05)$.

Local macrophage depletion inhibits corneal lymphangiogenesis. We next evaluated the effect of selective macrophage depletion in the cornea by subconjunctival injection of clodronate liposomes $(31,32)$. Macrophages that phagocytose clodronate liposomes rapidly die. Subconjunctival liposome injection on days $0,2,4$, and 6 was applied to eyes with centrally sutured corneas. Local depletion of macrophages nearly completely inhibited corneal lymphangiogenesis and HA (Figure 9); the areas of blood and lymph vessels in mice receiving clodronate were $11.3 \% \pm 5.8 \%$ and $10.8 \% \pm 2.5 \%$, respectively, compared with $42.3 \% \pm 11.3 \%$ and $38.8 \% \pm 4.7 \%$ for blood and lymph vessels, respectively, in PBS-treated controls $(P<0.01)$. There was no obvious direct effect of locally applied clodronate liposomes on preexisting limbal and pathological corneal blood or lymphatic vessels. These results demonstrate that macrophages, recruited to the site of injury by ligation of VEGFR1, are critical to inflammation-associated HA and lymphangiogenesis.

Macrophages in inflamed corneas express lymphangiogenic VEGF-C and -D. To directly assess whether macrophages recruited

\section{Figure 6}

Effect of VEGF-A on lymphangiogenesis in corneal micropocket assay. (A-C) Pellets $\left(^{*}\right)$ containing 200 ng VEGF-A always induced a robust hemangiogenic response ( $\mathbf{A}$, green; $\mathrm{Li}$, limbal vascular arcade [arrowhead]) and in 17 of 20 pellets in addition there was a mild to moderate lymphangiogenic response (red), which was significantly less compared with the hemangiogenic response (B). Panel $\mathbf{C}$ shows a representative and comparable effect by a VEGF-C pellet (200 ng). Magnification (A and C), $\times 100$.
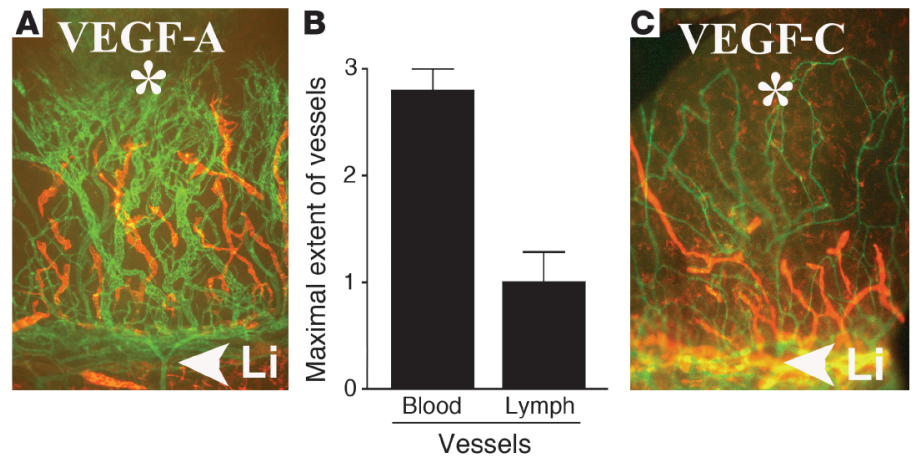

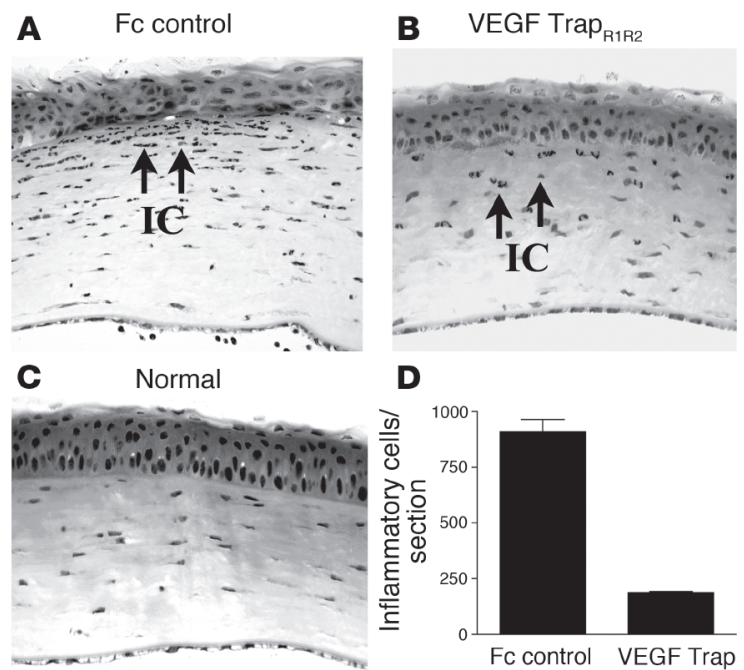

D
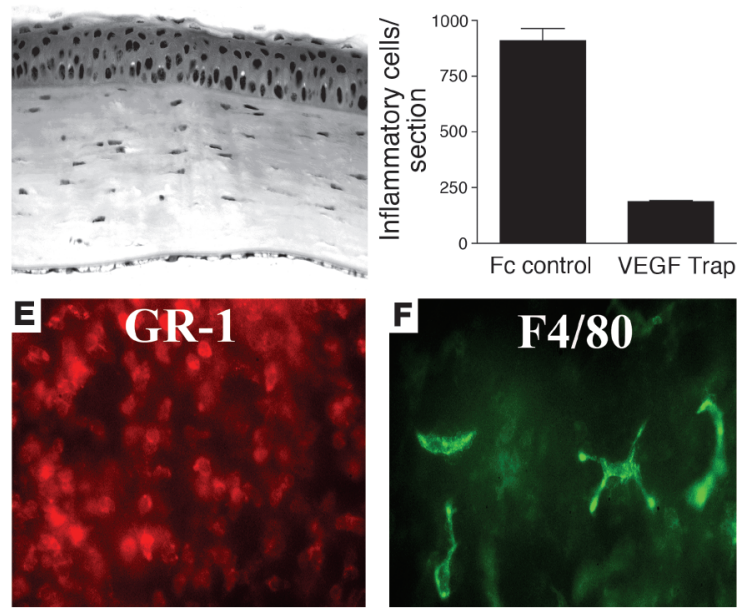

by VEGF-A into inflamed corneas are able to release lymphangiogenic growth factors VEGF-C and -D, we performed immunohistochemical studies in inflamed corneas 48 hours after suture placement using double labeling for VEGF-C/VEGF-D and the macrophage markers CD11b and F4/80. As depicted in Figure 10, this revealed that most $\mathrm{CD} 11 \mathrm{~b}^{+} \mathrm{F} 4 / 80^{+}$macrophages in the stroma were positive for VEGF-C and some were also positive for VEGF-D. To provide further support for the notion that mouse macrophages can express VEGF-C and -D, we performed RT-PCR studies on cultivated bone marrow-derived mouse macrophages. As shown in Figure 10, these macrophages were able to transcribe both VEGF-C and -D mRNA.

\section{Discussion}

The results we have obtained in the corneal model of inflammatory neovascularization allow two important conclusions to be drawn regarding the role of VEGF-A in blood and lymphatic vessel growth. First, endogenous VEGF-A can promote lymphangiogenesis, at least in the context of inflammatory forms of neovascularization. Second,

\section{Figure 8}

Bone marrow-derived cells mediate inflammation-associated lymphangiogenesis. (A-C) Depletion of bone marrow-derived cells induces a parallel inhibition of both $\mathrm{HA}$ and lymphangiogenesis in response to corneal inflammatory stimuli (blood vessels, green; lymphatic vessels, red). (A) Seven days after suture placement, control mice display parallel outgrowths of blood and lymphatic vessels from the limbal vascular arcade (left). (B and C) A single whole-body irradiation causes significant parallel inhibition of both HA and lymphangiogenesis. Inset in B shows a representative area of a normal limbal vascular arcade without vessel outgrowth. In $\mathbf{C}$, controls are compared to irradiated mice $(S+R x) ; P<0.05$. Magnification $(A$ and $B), \times 100$.

\section{Figure 7}

Anti-inflammatory effect of trapping VEGF-A. (A-C) Trapping of VEGF-A/ PIGF using the molecular cytokine trap VEGF Trap R1R2 $_{\text {significantly }}$ reduces the recruitment of inflammatory cells into the cornea in the suture-induced neovascularization model. One week after surgery, control mice treated with $\mathrm{Fc}$ protein ( $\mathrm{Fc}$ control) displayed a significant influx of inflammatory cells (IC and arrows) into the central corneal stroma (A). Trapping of VEGF-A significantly reduces this influx (B; and C, normal cornea). (D) Trapping of VEGF-A reduces stromal inflammatory cells by about $80 \%(P<0.01)$. ( $E$ and $\mathbf{F})$ The inflammatory cells found in the corneal stroma 7 days after suture placement and Fc treatment (controls) are overwhelmingly GR-1+ neutrophils (E, red) and less commonly, F4/80+CD11 $b^{+}$macrophages (F, green). Magnification, $\times 100$ (A-C) and $\times 400$ (E and F).

signaling via VEGFR1 on leukocytes, particularly monocytes/ macrophages, is a critical step in "immune amplification" of signals that promote pathological HA and lymphangiogenesis.

The present observations, that lymphangiogenesis and HA occur contemporaneously in $\mathrm{CNV}$ and that both responses are equally blocked by the selective inhibition of endogenous VEGF-A, appear to contradict the established notion that the ligation of VEGF-A to VEGFR2 induces solely HA, while interactions between VEGF-C/ VEGF-D and VEGFR3 discretely mediate lymphangiogenesis. Indeed, a substantial literature supports this essential dichotomy in the function of VEGF family proteins and their receptors. For example, when applied to differentiated chick chorioallantoic membrane (CAM), VEGF-A was found to stimulate HA, but not lymphangiogenesis, while VEGF-C induced only lymphangiogenesis (15). Interestingly, the VEGFR1-selective ligand PlGF was unable to induce either lymphangiogenesis or HA in the CAM assay. Similarly, in the corneal micropocket assay, VEGF-A was reported to induce HA but not lymphangiogenesis (11), and in several studies using adenoviral overexpression, VEGF-C consistently induced lymphangiogenesis, while VEGF-A did not (12-14). While these studies do demonstrate that VEGF-C/VEGFR3 and VEGF-A/ VEGFR2 interactions can induce pure lymphangiogenic and
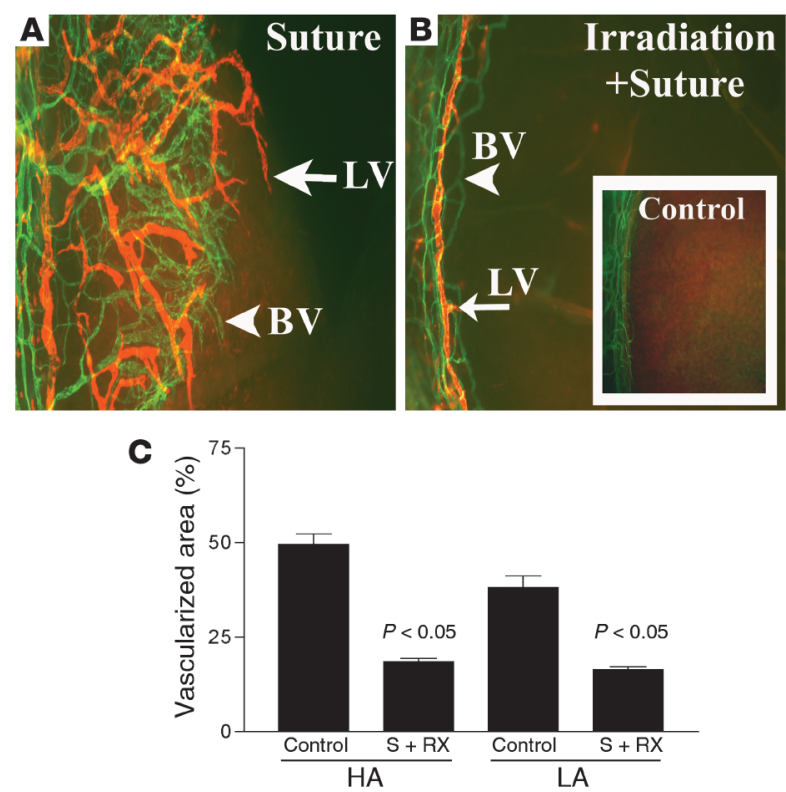
A
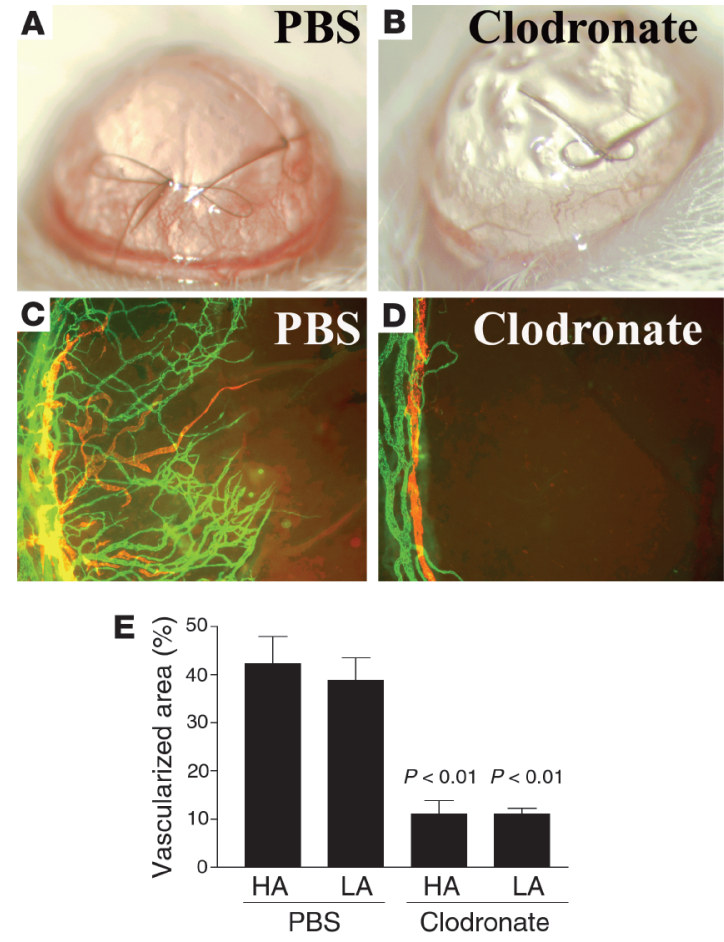

Figure 9

Macrophages are essential for pathological HA and lymphangiogenesis. (A and C) PBS-treated controls. (B and D) Mice that received subconjunctival clodronate liposomes. Magnification, $\times 100$ (C and D). (E) Depletion of macrophages inhibits both HA and lymphangiogenesis $(L A)$ in inflammatory neovascularization $(P<0.01)$. Magnification $(\mathbf{A}$ and $\mathbf{B}), \times 20$.

hemangiogenic responses, respectively, under certain conditions, more recent studies are beginning to show that this dichotomy is far from complete.

In fact, VEGF-C and -D possess dual lymphangiogenic and hemangiogenic properties $(2,9,10,36,37)$, and VEGFR3, while universally expressed by the lymphatic endothelium, is also expressed by vascular endothelial cells under some conditions, particularly during embryonic development and periods of active vessel remodeling, including that occurring in pathology $(34,37)$.

In contrast to VEGF-C and -D, there is comparatively little evidence to support the notion that VEGF-A might be involved in lymphangiogenesis. However, a recent molecular profiling study has shown that lymphatic endothelial cells can express VEGFR2 and that VEGF-A is as effective as VEGF-C in supporting their survival and promoting tube formation in vitro (16-19). Another recent study has demonstrated that adenoviral overexpression of VEGF- $A_{164}$ in the rabbit ear leads to the formation of "giant" lymphatic vessels (20). These studies raised the possibility that endogenous VEGF-A might, under some circumstances, play a role in promoting lymphangiogenesis - a possibility that we have confirmed in the present studies.

Specifically, we have demonstrated that (a) exogenous VEGF-A alone can induce lymphangiogenesis in the corneal pocket assay (different findings in a previous study [ref. 11] might be explained by the use of different mouse strains, amounts of VEGF-A and staining techniques); (b) lymphangiogenesis and HA occur contemporaneously in a corneal injury model of inflammatory neo- vascularization; (c) selective pharmacological neutralization of VEGF-A/PIGF completely inhibited both HA and lymphangiogenesis in this model due to primary inhibition of blood and lymphatic vessel formation rather than via accelerated regression; and (d) following corneal injury, both lymphangiogenesis and HA were equivalently reduced in transgenic mice that expressed only either VEGF-A $A_{164}$ or VEGF- $A_{188}(25,26)$. Taken together, these results demonstrate that endogenous VEGF-A plays a critical role in promoting lymphangiogenesis as well as HA, at least under certain pathophysiological conditions.

We next turned our attention to mechanisms that might explain the coordinate induction of HA and lymphangiogenesis in this model and the effective suppression of both responses by selective inhibition of VEGF-A. Here we noted that in addition to suppressing CNV, administration of VEGF Trap also significantly suppressed the inflammatory response that is induced by the placement of intrastromal corneal sutures. It is well established that VEGF-A is a potent monocyte chemoattractant and that this effect is mediated by ligation of VEGFR1 $(27,38,39)$. Thus, one likely scenario is that VEGF-A indirectly stimulates lymphangiogenesis in CNV by recruiting bone marrow-derived cells, particularly monocytes/macrophages, to the affected site and these cells in turn are the source of one or more lymphangiogenic factors. Activated leucocytes are know to express and secrete a large number of cytokines and other regulatory peptides and proteins, including VEGF-A $(31,40,41)$. Moreover, it has recently been
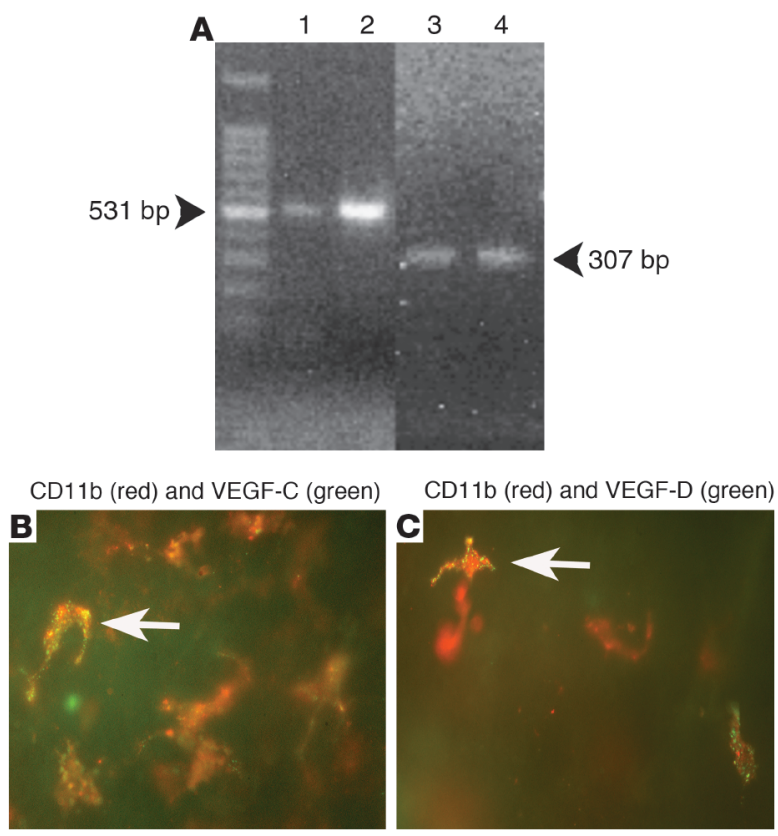

Figure 10

Macrophages in inflamed corneas express both VEGF-C and -D. (A) Cultivated, bone marrow-derived macrophages from BALB/c mice transcribe VEGF-C and -D mRNA 1 week after seeding. 1, VEGF-C positive control; 2, mouse bone marrow-derived macrophage VEGF-C; 3, VEGF-D positive control; 4: mouse bone marrow-derived macrophage VEGF-D. (B) Expression of VEGF-C (green) in red-stained CD11b+ macrophages in an inflamed cornea 48 hours after injury. (C) Expression of VEGF-D (green) in red-stained CD11b+ macrophages in an inflamed cornea 48 hours after injury. Arrows indicate a representative macrophage. Magnification (B and C), $\times 600$. 


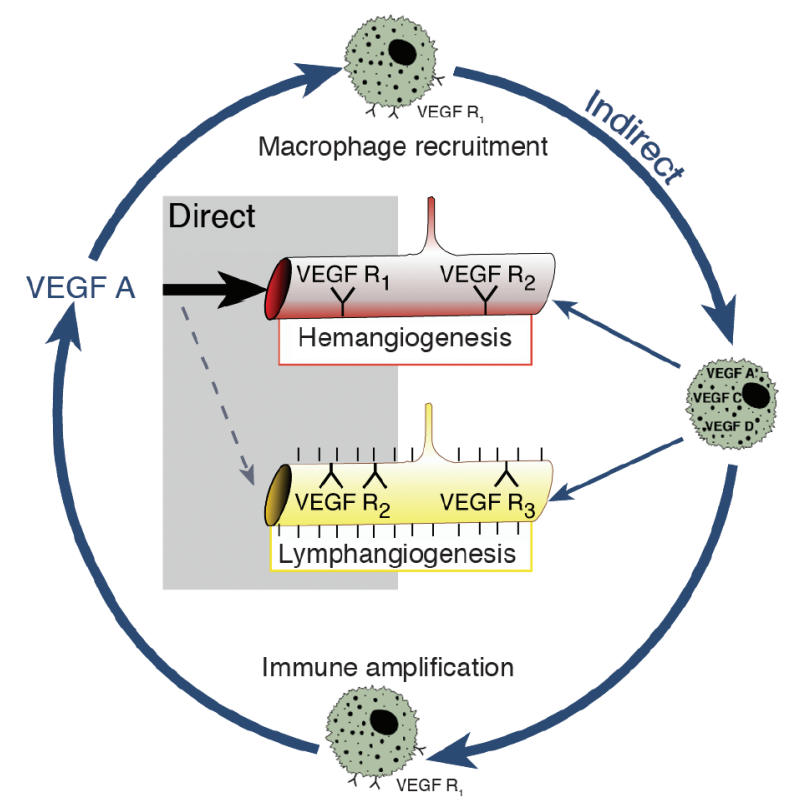

Figure 11

Proposed concept of an (indirect) lymphangiogenic role of VEGF-A via recruitment of bone marrow-derived macrophages, which in turn can release both hemangiogenic and lymphangiogenic growth factors. Macrophages seem to be important for immune amplification, leading to pathological HA and lymphangiogenesis.

shown that a subfraction of circulating VEGFR $3^{+} \mathrm{CD} 14^{+}$monocytes also strongly expresses VEGF-C and VEGF-D upon recruitment to peritumoral sites or in vitro stimulation (8). Moreover, $\mathrm{VEGF}^{-\mathrm{C}^{+}}$macrophages colocalize with new peritumoral lymph vessels, strongly suggesting a role for these cells in lymphangiogenesis $(8,42)$. Furthermore, it is known that proinflammatory cytokines, rather than hypoxia, upregulate VEGF-C expression (43) and that VEGF-C consequently is highly expressed in inflammatory conditions (44) suggesting even more strongly that VEGF-A-recruited macrophages upregulate VEGF-C/VEGF-D in response to corneal inflammatory cytokines. Indeed we have demonstrated here that $\mathrm{CD} 11 \mathrm{~b}^{+} \mathrm{F} 4 / 80^{+}$macrophages in the inflamed corneal stroma express VEGF-C (more than VEGF-D) and that bone marrow-derived mouse macrophages transcribe both VEGF-C and -D mRNA.

The results of the present study directly support the concept that VEGF-A-mediated recruitment of inflammatory cells by VEGFR1 ligation is an important step in the initiation of the lymphangiogenic response in CNV. Pharmacological neutralization of VEGF-A significantly inhibited recruitment of inflammatory cells into the cornea after suture placement. Moreover, systemic depletion of bone marrow-derived cells by irradiation significantly attenuated corneal lymphangiogenesis after an inflammatory stimulus. Furthermore, local depletion of macrophages using subconjunctival clodronate liposomes substantially inhibited lymphangiogenesis. Finally, macrophages in inflamed corneas expressed both lymphangiogenic VEGF-C and -D. Taken together, these findings provide strong evidence that macrophage recruitment is an essential mediator of the (indirect) lymphangiogenic effect of VEGF-A (Figure 11 depicts this concept). Here it is also important to note that macrophage depletion not only suppressed lymphangiogenesis following corneal injury but also effectively suppressed concomitant HA. This observation is consistent with a previous study showing that selective macrophage depletion inhibits pathological neovascularization in other disease models (45), supporting the notion that inflammation is also a requisite component of pathological HA mediated by VEGF-A $(45,46)$.

While VEGF-mediated recruitment of inflammatory cells clearly plays an important and apparently predominant role in promoting pathological neovascularization, it is quite likely that other, more direct actions of VEGF-A contribute to initiating both hemangiogenic and lymphangiogenic responses. For example, VEGF-A acts directly on vascular endothelium to upregulate the expression of adhesion molecules that promote leukostasis $(47,48)$. Likewise, rapid VEGF-mediated increases in the permeability of resident vessels and the consequent extravasation of serum proteins also serve to promote the subsequent formation of both blood and lymphatic vessels $(17,49)$. It is also possible that VEGF-A acts directly on VEGFR2 to promote the growth and organization of the lymphatic endothelium $(16,50)$. Final$1 y$, in addition to recruiting inflammatory cells that supply cytokines and growth factors to the site of injury, VEGF-A may also amplify angiogenic responses by recruiting VEGFR1-positive hematopoietic progenitor cells to the affected site and promoting their differentiation into vascular endothelium (for review see refs. 2, 51).

While our data strongly support the concept that recruitment of monocytes/macrophages by VEGF-A, through VEGFR1, is an early and essential step in an immune amplification cascade that leads to both inflammatory HA and lymphangiogenesis (see Figure 11), it is formally possible that the VEGFR1 ligand PIGF could also be partly responsible for promoting both corneal HA and lymphangiogenesis. Indeed, both VEGF TrapR1R2 and VEGF

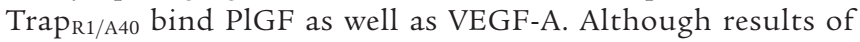
other studies indicate that PlGF can collaborate with VEGF-A in the stimulation of pathological HA $(51,52)$, three facts "argue against" the possibility that endogenous PlGF plays a significant role in promoting inflammatory lymphangiogenesis: (a) PIGF binds only to VEGFR1, while the lymphatic endothelium expresses only VEGFR2 and VEGFR3 (53); (b) overexpression of $\mathrm{AD}-\mathrm{PlGF}$ in the rabbit ear resulted in the formation of blood vessels, but in contrast to VEGF-A it did not cause lymphangiogenesis (20); and (c) in the present study, both lymphangiogenesis and HA were comparably reduced in VEGF-A isoform-deficient transgenic mice.

Currently, the most parsimonious mechanistic explanation for VEGF-A-mediated lymphangiogenesis in CNV is that VEGF-A promotes this response indirectly by binding to VEGFR1 and recruiting macrophages that secrete VEGF-C and/or VEGF-D at the site of injury. However, in a previous study, application of an exogenous VEGF-C isoform (156S) was unable to induce lymphangiogenesis in the cornea micropocket assay (in contrast, e.g., to the skin) (11, 54). Thus, while there is evidence that VEGFR3 signaling is necessary for corneal lymphangiogenesis (11), the hypothesis that VEGFR3-signalling is sufficient for the initiation of corneal lymphangiogenesis awaits experimental confirmation.

Inflammation is a common feature of diverse conditions characterized by pathological neovascularization, so it is quite possible that VEGF-A may play an important role in promoting lymphangiogenesis as well as abnormal HA in other disease states 
(42). If so, the present findings may have important ramifications for "antiangiogenesis" therapies currently in development for the treatment of a variety of diseases. As previously noted, a strong correlation exists between the degree of peritumoral inflammation and lymphangiogenesis in diverse types of human tumors (42). VEGF-A is highly expressed in most solid tumors and might serve to amplify lymphangiogenesis as well as HA in cancers by recruiting "lymphangiogenic" monocytes/macrophages. Thus, antiangiogenic strategies that target VEGF-A signaling might also prove effective in at least partially suppressing peritumoral lymphangiogenesis. In the context of corneal transplant rejection, recruitment of antigen-presenting cells into afferent lymphatic vessels is an essential step in the process by which the host immune response emerges to foreign transplant antigens. Therapeutic strategies aimed at suppressing newly outgrowing lymphatics should improve transplant survival by inhibiting allosensitization (C. Cursiefen and J.W. Streilein, unpublished observations). As immune rejection is the most important cause of corneal graft failure, our findings suggest that effective inhibitors of VEGF-A signaling have the potential to improve the survival of corneal transplants.

1. Folkman, J. 1995. Angiogenesis in cancer, vascular, rheumatoid and other disease. Nat. Med. 1:27-31.

2. Carmeliet, P. 2003. Angiogenesis in health and disease. Nat. Med. 9:653-660.

3. Kerbel, R., and Folkman, J. 2002. Clinical translation of angiogenesis inhibitors. Nat. Rev. Cancer. 2:727-739.

4. Stacker, S.A., Achen, M.G., Jussila, L., Baldwin, M.E., and Alitalo, K. 2002. Lymphangiogenesis and cancer metastasis. Nat. Rev. Cancer. 2:573-583.

5. He, Y., et al. 2002. Suppression of tumor lymphangiogenesis and lymph node metastasis by blocking vascular endothelial growth factor receptor 3 signaling. J. Natl. Cancer. Inst. 94:819-825.

6. Skobe, M., et al. 2001. Induction of tumor lymphangiogenesis by VEGF-C promotes breast cancer metastasis. Nat. Med. 7:192-198.

7. Padera, T.P., et al. 2002. Lymphatic metastasis in the absence of functional intratumor lymphatics. Science. 296:1883-1886.

8. Schoppmann, S.F., et al. 2002. Tumor-associated macrophages express lymphatic endothelial growth factors and are related to peritumoral lymphangiogenesis. Am. J. Pathol. 161:947-956.

9. Rissanen, T.T., et al. 2003. VEGF-D is the strongest angiogenic and lymphangiogenic effector among VEGFs delivered into skeletal muscle via adenoviruses. Circ. Res. 92:1098-1106.

10. Cao, Y., et al. 1998. Vascular endothelial growth factor $\mathrm{C}$ induces angiogenesis in vivo. Proc. Natl. Acad. Sci. U. S. A. 95:14389-14394.

11. Kubo, H., et al. 2002. Blockade of vascular endothelial growth factor receptor-3 signaling inhibits fibroblast growth factor-2-induced lymphangiogenesis in mouse cornea. Proc. Natl. Acad. Sci. U. S. A. 99:8868-8873.

12. Byzova, T.V., et al. 2002. Adenovirus encoding vascular endothelial growth factor-D induces tissuespecific vascular patterns in vivo. Blood. 99:4434-4442.

13. Enholm, B., et al. 2001. Adenoviral expression of vascular endothelial growth factor-C induces lymphangiogenesis in the skin. Circ. Res. 88:623-629.

14. Saaristo, A., et al. 2002. Adenoviral VEGF-C overexpression induces blood vessel enlargement, tortuosity, and leakiness but no sprouting angiogenesis in the skin or mucous membranes. FASEB J. 16:1041-1049.

15. Oh, S.J., et al. 1997. VEGF and VEGF-C: specific
Note added in proof. J. Wayne Streilein is deceased.

\section{Acknowledgments}

We thank our colleagues at the Schepens Eye Research Institute, especially J. Doherty for general support, J. Gu for help with histology, D. Pottle for help with confocal and immunofluorescent imaging, M. Ortega and T. Truong for help with mouse colony, and Peter Mallen for help with art work. We thank Ashique Rafique (Regeneron Pharmaceuticals Inc.) for help with the biochemical characterization of the VEGF Traps. This work was supported by Deutsche Forschungsgemeinschaft (Cu 47/1-1 and $\mathrm{Cu} 47 / 1-2)$ and by NIH grants EY10765 and CA45548. P.A. D'Amore is a Jules and Doris Stein Research to Prevent Blindness Professor.

Received for publication November 4, 2003, and accepted in revised form January 28, 2004.

Address correspondence to: Claus Cursiefen, Schepens Eye Research Institute, Harvard Medical School, 20 Staniford Street, Boston, Massachusetts 02114, USA; Phone: (617) 912-0100; Fax: (617) 912-0101; E-mail: cursiefen@vision.eri.harvard.edu. induction of angiogenesis and lymphangiogenesis in the differentiated avian chorioallantoic membrane. Dev. Biol. 188:96-109.

16. Podgrabinska, S., et al. 2002. Molecular characterization of lymphatic endothelial cells. Proc. Natl. Acad. Sci. U. S. A. 99:16069-16074.

17. Hirakawa, S., et al. 2003. Identification of vascular lineage-specific genes by transcriptional profiling of isolated blood vascular and lymphatic endothelial cells. Am. J. Pathol. 162:575-586.

18. Petrova, T.V., et al. 2002. Lymphatic endothelial reprogramming of vascular endothelial cells by the Prox-1 homeobox transcription factor. EMBO J. 21:4593-4599.

19. Jeltsch, M., et al. 1997. Hyperplasia of lymphatic vessels in VEGF-C transgenic mice. Science. 276:1423-1425.

20. Nagy, J.A., et al. 2002. Vascular permeability factor/vascular endothelial growth factor induces lymphangiogenesis as well as angiogenesis. J. Exp. Med. 196:1497-1506

21. Streilein, J.W., Yamada, J., Dana, M.R., and Ksander, B.R. 1999. Anterior chamber-associated immune deviation, ocular immune privilege, and orthotopic corneal allografts. Transplant. Proc. 31:1472-1475.

22. Cursiefen, C., et al. 2002. Lymphatic vessels in vascularized human corneas: immunohistochemical investigation using LYVE-1 and podoplanin. Invest. Ophthalmol. Vis. Sci. 43:2127-2135.

23. Chang, L., Kaipainen, A., and Folkman, J. 2002. Lymphangiogenesis new mechanisms. Ann. N. Y. Acad. Sci. 979:111-119.

24. Holash, J., et al. 2002. VEGF-Trap: a VEGF blocker with potent antitumor effects. Proc. Natl. Acad. Sci. U. S. A. 99:11393-11398.

25. Shima, D.T., et al. 1996. The mouse gene for vascular endothelial growth factor. Genomic structure, definition of the transcriptional unit, and characterization of transcriptional and post-transcriptional regulatory sequences. J. Biol. Chem. 271:3877-3883.

26. Stalmans, I., et al. 2002. Arteriolar and venular patterning in retinas of mice selectively expressing VEGF isoforms. J. Clin. Invest. 109:327-336.

27. Shen, H., et al. 1993. Characterization of vascular permeability factor/vascular endothelial growth factor receptors on mononuclear phagocytes. Blood. 81:2767-2773.

28. Barleon, B., et al. 1996. Migration of human monocytes in response to vascular endothelial growth factor (VEGF) is mediated via the VEGF receptor flt-1. Blood. 87:3336-3343.

29. Streilein, J.W., Bradley, D., Sano, Y., and Sonoda, Y. 1996. Immunosuppressive properties of tissues obtained from eyes with experimentally manipulated corneas. Invest. Ophthalmol. Vis. Sci. 37:413-424.

30. Banerji, S., et al. 1999. LYVE-1, a new homologue of the CD44 glycoprotein, is a lymph-specific receptor for hyaluronan. J. Cell. Biol. 144:789-801.

31. Van der Veen, G., et al. 1994. Prevention of corneal allograft rejection in rats treated with subconjunctival injections of liposomes containing dichloromethylene diphosphonate. Invest. Ophthalmol. Vis. Sci. 35:3505-3515.

32. Van Rooijen, N., and Sanders, A. 1994. Liposome mediated depletion of macrophages: mechanism of action, preparation of liposomes and applications. J. Immunol. Methods. 174:83-93.

33. Rutherford, M.S., and Schook, L.B. 1992. Differential immunocompetence of macrophages derived using macrophage or granulocytemacrophage colony-stimulating factor. J. Leukoc. Biol. 51:69-76.

34. Paavonen, K., Puolakkainen, P., Jussila, L., Jahkola, T., and Alitalo, K. 2000. Vascular endothelial growth factor receptor-3 in lymphangiogenesis in wound healing. Am. J. Pathol. 156:1499-1504.

35. Berse, B., Brown, L.F., Van de Water, L., Dvorak, H.F., and Senger, D.R. 1992. Vascular permeability factor (vascular endothelial growth factor) gene is expressed differentially in normal tissues, macrophages, and tumors. Mol. Biol. Cell. 3:211-220.

36. Alitalo, K., and Carmeliet, P. 2002. Molecular mechanisms of lymphangiogenesis in health and disease. Cancer Cell. 1:219-227.

37. Partanen, T.A., Alitalo, K., and Miettinen, M. 1999. Lack of lymphatic vascular specificity of vascular endothelial growth factor receptor 3 in 185 vascular tumors. Cancer. 86:2406-2412.

38. Clauss, M., et al. 1996. The vascular endothelial growth factor receptor Flt-1 mediates biological activities. Implications for a functional role of placenta growth factor in monocyte activation and chemotaxis. J. Biol. Chem. 271:17629-17634.

39. Hiratsuka, S., Minowa, O., Kuno, J., Noda, T., and Shibuya, M. 1998. Flt-1 lacking the tyrosine kinase domain is sufficient for normal development and angiogenesis in mice. Proc. Natl. Acad. Sci. U. S. A. 
95:9349-9354.

40. Freeman, M.R., et al. 1995. Peripheral blood T lymphocytes and lymphocytes infiltrating human cancers express vascular endothelial growth factor: a potential role for $\mathrm{T}$ cells in angiogenesis. Cancer Res. 55:4140-4145.

41. Taichman, N.S., Young, S., Cruchley, A.T., Taylor, P., and Paleolog, E. 1997. Human neutrophils secrete vascular endothelial growth factor. J. Leukoc. Biol. 62:397-400.

42. Schoppmann, S.F., et al. 2001. Inflammatory stromal reaction correlates with lymphatic microvessel density in early-stage cervical cancer. Anticancer Res. 21:3419-3423.

43. Paavonen, K., et al. 2002. Vascular endothelial growth factors C and D and their VEGFR- 2 and 3 receptors in blood and lymphatic vessels in healthy and arthritic synovium. J. Rheumatol. 29:39-45.

44. Ristimaki, A., Narko, K., Enholm, B., Joukov, V., and Alitalo, K. 1998. Proinflammatory cytokines regulate expression of the lymphatic endothelial mitogen vascular endothelial growth factor-C. J. Biol. Chem. 273:8413-8418.

45. Ishida, S., et al. 2003. VEGF164-mediated inflammation is required for pathological, but not physiological, ischemia-induced retinal neovascularization. J. Exp. Med. 198:483-489.

46. Fromer, C.H., and Klintworth, G.K. 1975. An evaluation of the role of leukocytes in the pathogenesis of experimentally induced corneal vascularization. II. Studies on the effect of leukocytic elimination on corneal vascularization. Am. J. Pathol. 81:531-544.

47. Melder, R.J., et al. 1996. During angiogenesis, vascular endothelial growth factor and basic fibroblast growth factor regulate natural killer cell adhesion to tumor endothelium. Nat. Med. 2:992-997.

48. Lu, M., et al. 1999. VEGF increases retinal vascular ICAM-1 expression in vivo. Invest. Ophthalmol. Vis. Sci. 40:1808-1812.

49. Boardman, K.C., and Swartz, M.A. 2003. Intersti- tial flow as a guide for lymphangiogenesis. Circ. Res. 92:801-808.

50. Gerhardt, H., et al. 2003. VEGF guides angiogenic sprouting utilizing endothelial tip cell filopodia. J. Cell. Biol. 161:1163-1177.

51. Luttun, A., Tjwa, M., and Carmeliet, P. 2002. Placental growth factor (PlGF) and its receptor Flt-1 (VEGFR-1): novel therapeutic targets for angiogenic disorders. Ann. N. Y. Acad. Sci. 979:80-93.

52. Luttun, A., et al. 2002. Revascularization of ischemic tissues by PlGF treatment, and inhibition of tumor angiogenesis, arthritis and atherosclerosis by anti-Flt1. Nat. Med. 8:831-840. 53. Karkkainen, M.J., and Petrova, T.V. 2000. Vascular endothelial growth factor receptors in the regulation of angiogenesis and lymphangiogenesis. Oncogene. 19:5598-5605.

54. Veikkola, T., et al. 2001. Signalling via vascular endothelial growth factor receptor-3 is sufficient for lymphangiogenesis in transgenic mice. $E M B O J$. 20:1223-1231. 